Article

\title{
Qualitative and Quantitative Analysis of Different Rhodiola rosea Rhizome Extracts by UHPLC-DAD-ESI-MS ${ }^{n}$
}

\author{
Fabian Alperth ${ }^{1}$, Ivana Turek ${ }^{1}$, Sandra Weiss ${ }^{1}$, Dietmar Vogt ${ }^{2}$ and Franz Bucar ${ }^{1, *(D)}$ \\ 1 Institute of Pharmaceutical Sciences, University of Graz, Universitätsplatz 4, 8010 Graz, Austria; \\ fabian.alperth@edu.uni-graz.at (F.A.); iva.tureq@outlook.com (I.T.); sandraweiss2@gmx.at (S.W.) \\ 2 Phytagoras, 9020 Klagenfurt, Austria; dietmar.vogt1@gmail.com \\ * Correspondence: franz.bucar@uni-graz.at; Tel.: +43-316-380-5531
}

Received: 14 February 2019; Accepted: 26 March 2019; Published: 29 March 2019

\begin{abstract}
Rhodiola rosea has been used in folk medicine as ethanolic macerates for a long time. This study aims to provide a quantitative and qualitative analysis and comparison of different ethanolic Rhodiola rosea rhizome macerates $(35 \%, 70 \%$, and $96 \% v / v)$ and accelerated solvent extraction (ASE) extracts prepared with $85 \%$ methanol, in order to shed light on the effectivity of different extraction methods. Extract samples were analyzed by UHPLC-DAD-ESI-MS ${ }^{n}$ on a ZORBAX SB-C18 column $(100 \times 2.1 \mathrm{~mm}, 1.8 \mu \mathrm{m})$ with a mobile phase consisting of water + $0.1 \%$ formic acid and acetonitrile. Qualitative analysis lead to the tentative identification of 18 compounds: Two cyanogenic glycosides (rhodiocyanoside A, lotaustralin), three phenylethanoids (salidroside, viridoside, 2-phenylethyl-vicianoside), two procyanidin and catechin derivatives (epigallocatechin-epigallocatechin gallate, epigallocatechin-3-O-gallate), five phenylpropanoids (cinnamyl alcohol, rosarin, rosavin, rosin, cinnamyl-(6'-O- $\beta$-D-xylopyranosyl)-O- $\beta$-glucopyranoside), two monoterpene alcohols (rhodioloside $\mathrm{E}$, rosiridin) and four flavonols (rhodionidin, rhodiosin, rhodionin, kaempferol). Quantity was determined for salidroside, cinnamyl alcohol and its three major glycosides (rosarin, rosavin, rosin), as well as three flavonols (rhodionidin, rhodiosin, rhodionin). Methanolic ASE proved to be the superior extraction method for different substance groups. For macerates, high ethanol content increased yield and lowered hydrolysis of glycosides during extraction, but ethanolic macerates still showed low reproducibility and high fluctuations in quantity of marker compounds salidroside and rosavins, as well as flavonols. Rhodiola rosea rhizomes of wild origins seemed to underly great variability in chemical composition dependent on grow site.
\end{abstract}

Keywords: Rhodiola rosea; salidroside; cinnamyl alcohol glycosides; flavonols; ASE; UHPLC-MS

\section{Introduction}

Rhodiola rosea L. (Sedum roseum (L.) Scop.) is a herbaceous plant belonging to the family Crassulaceae. It grows up to $70 \mathrm{~cm}$ in height, has succulent leaves, and forms thick rhizomes [1]. Its common names include golden root and arctic root, among others [2]. The plant is indigenous to arctic regions of eastern Siberia, but wild populations can also be found on rocky terrain, sea-cliffs and mountains in northern and central Europe, Alaska, or Canada [2-4]. For centuries, preparations of Rhodiola rosea have found use in Altai folk medicine as a tonic and for treatment of several conditions, in form of infusions and tinctures [1]. References for use in Scandinavia and Iceland date back to the $18^{\text {th }}$ century, with different indications like headache, diarrhea, skin conditions, swellings and as tonic. Still today, the indigenous Sami of northern Scandinavia are reported to chew on rhizomes during long journeys, for its adaptogenic properties [3]. Rhodiola rosea extracts have officially been recommended in Russia as 
a stimulant and to milden different psychological deficiencies since 1969. In Sweden, tablets containing Rhodiola rosea extract SHR-5 have been on the market since 1985 [3].

Extracts of Rhodiola rosea have been pharmacologically tested for various effects. Among others, they show protection against oxidative agents in human erythrocytes in vitro [5,6], anti-fatigue effects during and after physical exercise in rats [7], improved resistance to restraint stress in rabbits [8], increased resistance of rats to mild stressors over an extended period of time [9], monoamino oxidase A and B inhibition in vitro [10], anti-inflammatory effects in rats [11], and $\alpha$-amylase and $\alpha$-glucosidase inhibitory activities [12].

About 140 compounds have been found in the underground organs of Rhodiola rosea, comprising monoterpene alcohols, cyanogenic glycosides, aryl glycosides, phenylethanoids, phenylpropanoids, flavonoids, proanthocyanidins, as well as gallic acid derivatives [3]. Compounds thought to be responsible for activities are phenylethanoids salidroside, and its aglycone tyrosol, as well as a group of phenylpropanoids, namely the cinnamyl alcohol glycosides rosin, rosarin, and rosavin [13]. Pharmacological studies investigating isolated compounds are most abundant for salidroside. Some reported effects are attenuation of rat hippocampal neuron cell-death, induced by glutamate in vitro [14], cardioprotective activity against hypoxia-induced cell-death in rat cardiomyocytes [15], and increased glucose uptake in rat myoblast cells [16]. Flavonoids from Rhodiola rosea have also been pharmacologically investigated. Rhodiosin and rhodionin—two flavonols-showed antioxidant activity [17] and lipase inhibition in mice [18]. Several flavonoids have shown antiviral effects through neuraminidase inhibition in vitro [19].

Occurrence of salidroside is a group characteristic for all Rhodiola species, cinnamic alcohol and its glycosides rosavin, rosarin, and rosin are described as marker compounds for Rhodiola rosea [1]. The quantification of salidroside, rosavin and rosarin is a key factor in quality assessment of formulations containing Rhodiola rosea [20].

Our study aims to provide a qualitative and quantitative analysis, as well as comparison, of different Rhodiola rosea rhizome extracts, prepared by maceration with different concentrations of ethanol and accelerated solvent extraction with methanol. Maceration with ethanol represents a preparation method for Rhodiola rosea that has been used in traditional medicine for a long time [1]. In combination with the use of fresh plant material it is the easiest preparation method utilizing a nontoxic solvent. In accelerated solvent extraction, lower extraction time and increased yield through elevated temperature and pressure, as well as the use of more polar solvent methanol, optimize the extraction process, in particular for the fast and reproducible extraction of glycosides. Higher extraction temperatures result in better solubility of the analytes, higher diffusion rates and lower solvent viscosity. The effectivity of simple maceration versus more modern instrument-based accelerated solvent extraction has not yet been shown in a comparative study.

\section{Materials and Methods}

\subsection{Solvents and Reference Substances}

All solvents used were of analytical grade or higher, purchased from VWR Prolabo Chemicals (Radnor, PA, USA) and Merck KGaA (Darmstadt, Germany). Reference substances salidroside (Sigma-Aldrich, St. Louis, Missouri, USA) and cinnamyl alcohol (Carl Roth KG, Karlsruhe, Germany) had a purity of over 98\%. For kaempferol-7-neohesperoside $\cdot \mathrm{H}_{2} \mathrm{O}$ (Carl Roth KG) purity was determined via peak integration in UV-total scan chromatograms $(190-500 \mathrm{~nm})$ to be $85.1 \%$ and was considered during quantitative calculations.

\subsection{Plant Material}

Rhodiola rosea rhizomes were obtained at altitudes of $2265 \mathrm{~m}$ in the region of High Tauern (Goldberg Group, Fragant, Striedental) and $1918 \mathrm{~m}$ in the Gurktal Alps (Nock Mountains, Falkert) (all Carinthia, Austria), under permission of the Carinthian district authorities of Spittal an der Drau and Feldkirchen, 
issued in November and December of 2014 with decrees SP3-NS-2459/2014 and FE3-NS-1921/2014. Samples were collected from May to October 2015, with a collection date before, during and after the flowering season. Identification was done by Dietmar Vogt; rhizome vouchers were deposited at the Institute of Pharmaceutical Sciences, University of Graz (Graz, Austria).

\subsection{Preparation of Extracts}

Separate fresh rhizome samples of each collection were extracted by maceration with $38 \%, 70 \%$ and $96 \%(v / v)$ ethanol for three weeks, every batch consisting of $20 \mathrm{~g}$ plant material in $100 \mathrm{~g}$ of solvent. Furthermore, fresh plant material was cut and freeze-dried for two days, using a VirTis Sentry freeze dryer (SP Scientific, Warminster, PA, USA), before being powdered in a blender. A total of $1 \mathrm{~g}$ of powdered plant material from each collection date was then extracted by accelerated solvent extraction (ASE) with $85 \%$ methanol at a temperature of $60^{\circ} \mathrm{C}$ and the pressure at $68.9 \mathrm{bar}$, using a Dionex ASE 200 Accelerated Solvent Extractor (Thermo Fisher Scientific, Waltham, MA, USA). Extraction consisted of three cycles, with $5 \mathrm{~min}$ of heating and $5 \mathrm{~min}$ of static extraction, which yielded about $20 \mathrm{ml}$ of extract. ASE extracts were filled up to $25 \mathrm{~mL}$ with $85 \%$ methanol to represent $1 \mathrm{~g}$ of freeze-dried plant material in $25 \mathrm{~mL}$ of solvent.

\subsection{UHPLC-DAD-ESI-MS ${ }^{n}$ Instrumentation and Methods}

Ultra-high performance liquid chromatography (UHPLC) analysis was conducted using a Dionex Ultimate 3000 RS system (Thermo Fisher Scientific), consisting of pump, autosampler, column compartment and diode array detector (DAD). Separation was performed on a ZORBAX SB-C18 Rapid Resolution HD analytical column (Agilent, Santa Clara, CA, USA), $100 \times 2.1 \mathrm{~mm}, 1.8 \mu \mathrm{m}$. The mobile phase was made up of water $+0.1 \%$ formic acid (A) and acetonitrile (B). Each run started at $2 \%$ B, increasing to $22 \% \mathrm{~B}$ at $13.33 \mathrm{~min}, 70 \% \mathrm{~B}$ at $22.22 \mathrm{~min}$, then dropping back to $2 \% \mathrm{~B}$ at $22.66 \mathrm{~min}$, and keeping this composition steady until finishing at $28 \mathrm{~min}$. The column temperature was set to $40^{\circ} \mathrm{C}$ and flow rate was set to $0.450 \mathrm{ml} / \mathrm{min}$. Injection volume was $2.5 \mu \mathrm{L}$ for ASE extracts and $5.0 \mu \mathrm{L}$ for macerates. All samples were filtered through a Teflon filter with $0.45 \mu \mathrm{m}$ mesh to rid the solution of suspended particles before UHPLC analysis. DAD-UV detection was set to a wavelength range of 200 to $400 \mathrm{~nm}$. Mass spectrometric (MS) detection was achieved with an LTQ XL linear ion-trap mass spectrometer equipped with an electrospray ionization (ESI) ion source (all Thermo scientific). Mass spectra were recorded in negative ion mode with m/z ranging from 50 to $2000 \mathrm{amu}$. Mass spectral conditions were set as follows: Source voltage $3.5 \mathrm{kV}$ (ESI neg); capillary temperature $350^{\circ} \mathrm{C}$; source temperature $300^{\circ} \mathrm{C}$; sheath gas flow 40 arb (arbitrary units), auxiliary gas flow 10 arb.

\subsection{Validation}

Quantification of phenolics in extracts was performed after validation of UHPLC methods using reference substances cinnamyl alcohol, salidroside and kaempferol-7-neohesperoside of known concentrations.

\subsubsection{Linearity}

Assessment of linearity in detector response for UHPLC methods was conducted for the three reference substances in a working range of 5-700 $\mu \mathrm{g} / \mathrm{mL}$. Samples were prepared and measured at concentrations of $5,10,50,100,200,500$ and $700 \mu \mathrm{g} / \mathrm{mL}$ in $85 \%$ methanol and resulted in individual calibration curves. Linear regression showed correlation coefficients of $\left(R^{2}\right)>0.9984,0.9995$ and 0.9993 for cinnamyl alcohol, salidroside and kaempferol-7-neohesperoside, respectively. 


\subsubsection{Precision}

Intra-day precision was determined by analysing six replicates of the same extract sample solution and comparing resulting peak areas for cinnamyl alcohol. Relative standard deviation (RSD) was found to be $0.5 \%$.

\subsubsection{Accuracy}

Accuracy was examined for the three reference substances at concentrations within the working range in duplicate. Reference samples were prepared as 75, 300 and $600 \mu \mathrm{g} / \mathrm{mL}$ in $85 \%$ methanol and quantities calculated according to predetermined calibration curves. Comparison with target values resulted in accuracies as shown in Table 1.

Table 1. Accuracies (\%) determined for reference standards at given concentrations.

\begin{tabular}{cccc}
\hline Concentration $(\mu \mathrm{g} / \mathrm{mL})$ & Accuracy Cinnamyl Alcohol & Accuracy Salidroside & $\begin{array}{c}\text { Accuracy } \\
\text { Kaempferol-7-Neohesperoside }\end{array}$ \\
\hline 75 & 98.9 & 95.1 & 109.7 \\
600 & 104.5 & 91.2 & 93.1 \\
& 98.1 & 90.5 & 95.0 \\
\hline
\end{tabular}

\section{Results}

\subsection{UHPLC-DAD-ESI-MS Analysis}

\subsubsection{Identification of Phenolic Constituents}

Qualitative results were obtained by interpreting mass spectrometric and DAD-UV data in the UHPLC chromatographic profile of the ASE extract of Rhodiola rosea rhizomes, collected during the flowering season in the Gurktal Alps. Constituents were tentatively identified by comparison with literature data describing compounds previously found in in the plant. Since the phenolic profile of Rhodiola rosea had already been intensively researched and molecular mass for inquired substances matched, there was reasonably little margin of error in this approach. Results and references for the occurrence of constituents in Rhodiola rosea can be found in Table 2. 
Table 2. Qualitative analysis of Rhodiola rosea rhizome accelerated solvent extraction (ASE) extract from collection site Gurktal Alps; rhizomes were collected during flowering season.

\begin{tabular}{|c|c|c|c|c|c|c|}
\hline No & RT (min) & [M-H] (m/z) & $\mathrm{MS}^{\mathrm{n}}(\mathrm{m} / \mathrm{z})$ & $\mathrm{UV} \lambda_{\max }(\mathrm{nm})$ & Identification & Reference \\
\hline 1 & 3.09 & 258 & $\begin{array}{l}\operatorname{MS}^{2}[304]^{1}: 258,179,161 \\
\operatorname{MS}^{3}[179]: 161,143,131,119,89\end{array}$ & 209 & Rhodiocyanoside A & [21] \\
\hline 2 & 4.08 & 260 & $\begin{array}{l}\operatorname{MS}^{2}[306]^{1}: 260,188,161 \\
\operatorname{MS}^{3}[260]: 188,161\end{array}$ & 205 & Lotaustralin & [22] \\
\hline 3 & 4.85 & 299 & $\begin{array}{l}\operatorname{MS}^{2}[345]^{1}: 299 \\
\text { MS }^{3}[299]: 179,161,143,131,119,113,101,89\end{array}$ & 222,278 & Salidroside & [23-25] \\
\hline 4 & 5.62 & 761 & $\begin{array}{l}\operatorname{MS}^{2}[761]: 635,609,593,592,575,483,423 \\
\operatorname{MS}^{3}[423]: 405,299,283,243\end{array}$ & 268 & $\begin{array}{l}\text { Epigallocatechin-epigallocatechin } \\
\text { gallate }\end{array}$ & [26] \\
\hline 5 & 6.56 & 313 & $\begin{array}{l}\mathrm{MS}^{2}[359]^{1}: 313 \\
\mathrm{MS}^{3}[313]: 151\end{array}$ & 226,271 & Viridoside & {$[24,25]$} \\
\hline 6 & 7.68 & 457 & $\begin{array}{l}\operatorname{MS}^{2}[457]: 331,305,169 \\
\operatorname{MS}^{3}[169]: 125\end{array}$ & 277 & Epigallocatechin-3-O-gallate & [26] \\
\hline 7 & 8.32 & 415 & $\begin{array}{l}\text { MS}^{2}[461]^{1}: 415 \\
\text { MS }^{3}[415]: 191,149,131\end{array}$ & & 2-Phenylethyl-vicianoside & {$[24,25]$} \\
\hline 8 & 9.40 & 609 & 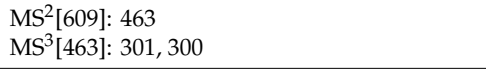 & $277,329,375$ (sh) & Rhodionidin & [27] \\
\hline 9 & 10.20 & 427 & $\begin{array}{l}\operatorname{MS}^{2}[473]^{1}: 427,293 \\
\operatorname{MS}^{3}[427]: 293,149\end{array}$ & 253 & Rosarin & [23-25] \\
\hline 10 & 10.42 & 465 & $\begin{array}{l}\operatorname{MS}^{2}[511]{ }^{1}: 465 \\
\operatorname{MS}^{3}[465]: 333,293,191,149\end{array}$ & & Rhodioloside E & [28] \\
\hline 11 & 10.53 & 427 & $\begin{array}{l}\operatorname{MS}^{2}[473]^{1}: 427,293 \\
\operatorname{MS}^{3}[427]: 293,149\end{array}$ & 253 & Rosavin & {$[23,25]$} \\
\hline 12 & 10.70 & 295 & $\begin{array}{l}\text { MS }^{2}[341]^{1}: 295,179,161 \\
\text { MS }^{3}[179]: 135\end{array}$ & 253 & Rosin & [23-25] \\
\hline 13 & 10.82 & 427 & $\begin{array}{l}\operatorname{MS}^{2}[473]^{1}: 427,293 \\
\operatorname{MS}^{3}[427]: 293,149\end{array}$ & 245 & $\begin{array}{l}\text { Cinnamyl-(6'-O- } \beta \text {-D-xylopyranosyl)- } \\
\text { O- } \beta \text {-glucopyranoside }\end{array}$ & {$[23,25]$} \\
\hline 14 & 11.06 & 331 & $\begin{array}{l}\operatorname{MS}^{2}[377] \\
\operatorname{MS}^{3}[331]: 179,161,143,113\end{array}$ & $192,264(\mathrm{sh})$ & Rosiridin & [23-25] \\
\hline 15 & 13.50 & -2 & & 205,253 & Cinnamyl alcohol & [29] \\
\hline 16 & 14.21 & 609 & $\begin{array}{l}\operatorname{MS}^{2} \text { [609]: } 301 \\
\operatorname{MS}^{3}[301]: 301,283,255,229,211,201\end{array}$ & $277,333,385$ & Rhodiosin & [17] \\
\hline 17 & 14.56 & 447 & $\begin{array}{l}\text { MS }^{2}[447]: 301 \\
\text { MS }^{3}[301]: 301,283,255,229,211,201\end{array}$ & $277,332,385$ & Rhodionin & [17] \\
\hline 18 & 16.78 & 285 & $\begin{array}{l}\operatorname{MS}^{2}[285]: 285,267,229,213,185,169,151 \\
\text { MS }^{3}[151]: 107\end{array}$ & 267,368 & Kaempferol & [27] \\
\hline
\end{tabular}

${ }^{1}$ Fragmentation of $\left([\mathrm{M}-\mathrm{H}]^{-}+\mathrm{HCOOH}\right)$ in $\mathrm{MS}^{2}{ }^{2}$ No ionization in ESI-MS $(-)$. 
Eighteen substances were tentatively identified by comparing negative molecular ions detected during UHPLC-DAD-ESI-MS ${ }^{n}$ analysis with molecular mass data for constituents of Rhodiola rosea, as found in literature. In negative ionization mode, negative molecular ions are indicated by $\mathrm{m} / \mathrm{z}$ values that equal $[\mathrm{M}-\mathrm{H}]^{-}$, the molecular mass of the compound minus one proton. In several peaks formic acid adducts were detected as $\left([\mathrm{M}-\mathrm{H}]^{-}+\mathrm{HCOOH}\right)$, which fully dissociated into $[\mathrm{M}-\mathrm{H}]^{-}$in $\mathrm{MS}^{2}$.

Two cyanogenic glycosides were tentatively identified as rhodiocyanoside A (1) and lotaustralin (2), both containing glucopyranoside as sugar moiety.

Three phenylethanoids were found. Salidroside (3) and viridoside (5) again contain glucopyranoside as O-glycosidic linked group, in 2-phenylethyl-vicianoside (7) the aglycone is bound to arabinopyranosyl-glucopyranoside. Two gallic acid derivatives present in the sample were interpreted as epigallocatechin-epigallocatechin gallate (4) and epigallocatechin-3-O-gallate (6).

Among five phenylpropanoids, cinnamyl alcohol (15) was identified by comparing UV data showing maxima at 206 and $247 \mathrm{~nm}$, as this substance did not ionize and therefore yielded no molecular ion. Subsequently, rosarin (9), rosavin (11), rosin (12) and compound (13), cinnamyl-(6'-O- $\beta$-D-xylopyranosyl)-O- $\beta$-glucopyranoside, were found to be glycosides of (15). Their sugar moieties consist of arabinopyranosyl-glucopyranoside for (9), arabinofuranosyl-glucopyranoside for (11), glucopyranoside for (12) and xylopyranosyl-glucopyranoside for (13).

Two monoterpene alcohols were assigned as rhodioloside E (10), containing arabinopyranosyl-glucopyranoside, and rosiridin (14) containing glucopyranoside.

Four flavonols were tentatively identified as rhodionidin (8), rhodiosin (16), rhodionin (17) and kaempferol (18). The first three are herbacetin glycosides with rhamnopyranoside and glucopyranoside (8), glucopyranosyl-rhamnopyranoside (16) and rhamnopyranoside (17) as sugar moieties, respectively. Kaempferol (18) is an aglycone. Rhodionidin (8) showed as the negative molecular ion [M-H] $]^{-}$at $609 \mathrm{~m} / \mathrm{z}$ in $\mathrm{MS}^{1}$. A fragment of $463 \mathrm{~m} / \mathrm{z}$ after loss of $146 \mathrm{u}$ is consistent with a glycosidically bound deoxyhexose like rhamnopyranoside [30]. Subsequent loss of a hexose is evident by $-162 \mathrm{u}$ in $\mathrm{MS}^{3}$, to form the negative aglycone ion at $301 \mathrm{~m} / \mathrm{z}$, in accordance with herbacetin having a molecular weight of 302. Rhodiosin (16) also appeared as negative molecular ion at $609 \mathrm{~m} / \mathrm{z}$ in $\mathrm{MS}^{1}$. Here, the loss of $308 \mathrm{u}$ already occurs in $\mathrm{MS}^{2}$ again giving the negative aglycone ion at $301 \mathrm{~m} / \mathrm{z}$. This is consistent with the O-glycosidic bound diglycoside of rhodiosin. Rhodionin (17) was indicated by a negative molecular ion of $447 \mathrm{~m} / \mathrm{z}$ in $\mathrm{MS}^{1}$. As with (8), loss of $146 \mathrm{u}$ indicates rhamnopyranoside in $\mathrm{MS}^{2}$ to form the aglycone ion at $301 \mathrm{~m} / \mathrm{z}$. Kaempferol (18) was detected as a negative aglycone ion of $285 \mathrm{~m} / \mathrm{z}^{\text {in }} \mathrm{MS}^{1}$, with a prominent fragment ion at $151 \mathrm{~m} / \mathrm{z}$ in $\mathrm{MS}^{2}$. This appears to be the ${ }^{1,3} \mathrm{~A}^{-}$fragment ion, formed after ring bond cleavage in the C-ring. Structures of proposed compounds are given in Figures 1-5.

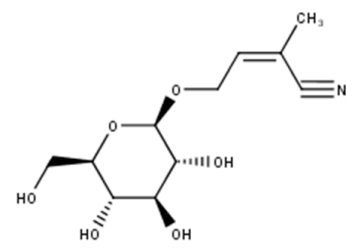

Rhodiocyanoside A (1)

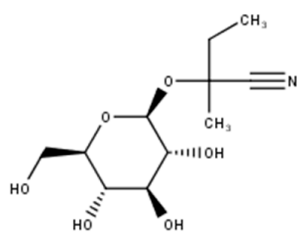

Lotaustralin (2)

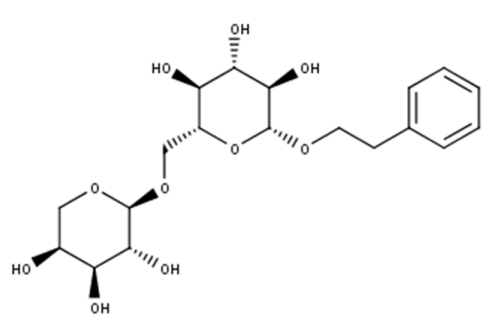

2-Phenylethyl-vicianoside (7)

Figure 1. Structures of proposed cyanogenic glycosides $(\mathbf{1}, \mathbf{2})$ and phenylethanoids $(3,5,7)$. 
<smiles>O=C(O[C@H]1Cc2c(O)cc(O)cc2O[C@H]1c1cc(O)c(O)c(O)c1)c1cc(O)c(O)c(O)c1</smiles>

Figure 2. Structure of proposed compound epigallocatechin-3-O-gallate (6).

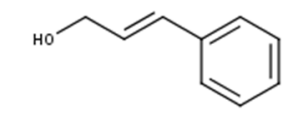

Cinnamyl alcohol (15)

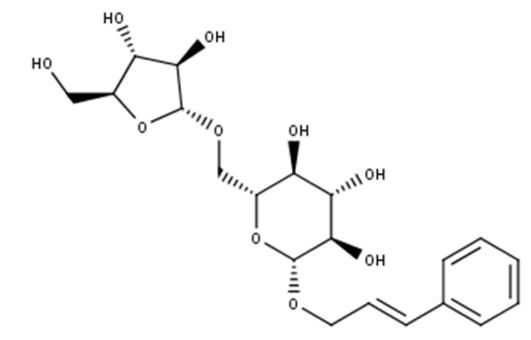

Rosarin (9)

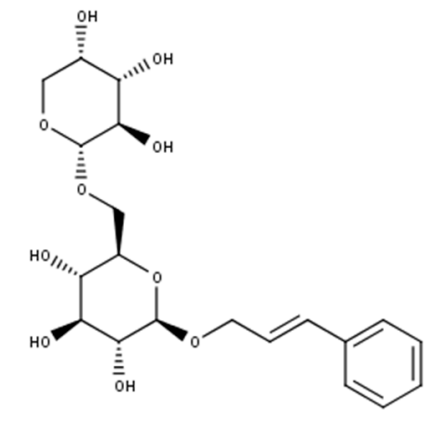

Rosavin (11)
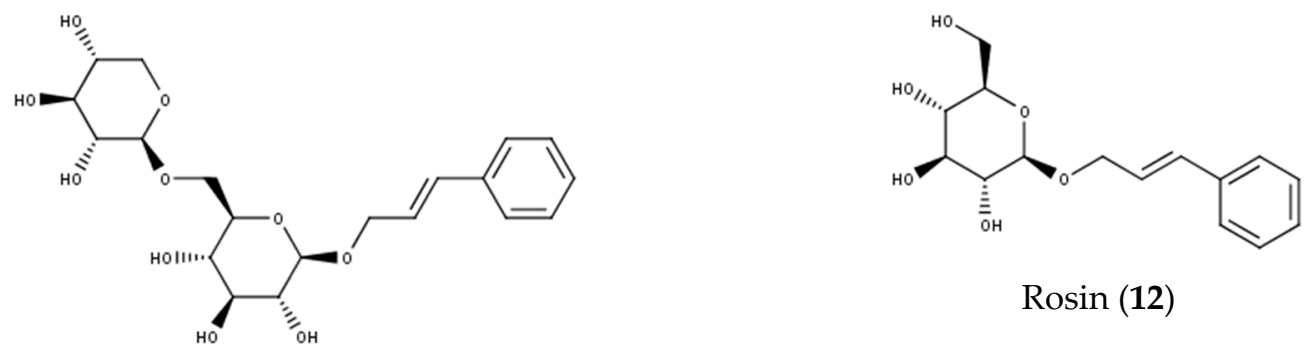

$\operatorname{Rosin}(\mathbf{1 2})$

Cinnamyl-(6'-O- $\beta$-D-xylopyranosyl)-O- $\beta$ glucopyranoside (13)

Figure 3. Structures of proposed phenylpropanoids $(9,11-13,15)$.

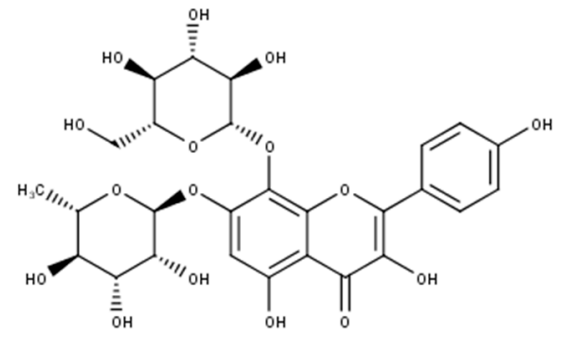

Rhodionidin (8)<smiles>Cc1cc(C)c2c(=O)c(O)c(-c3ccc(I)cc3)oc2c1C</smiles>

Rhodionin (17)

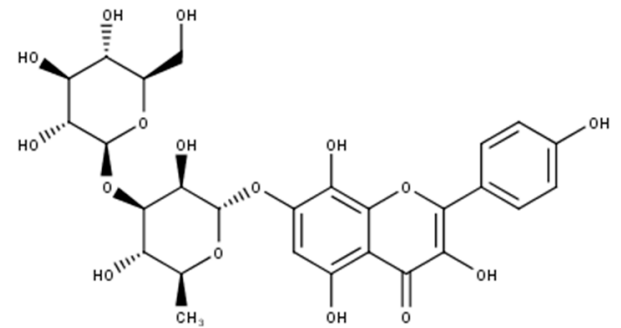

Rhodiosin (16)<smiles>Cc1cc(O)c2c(=O)c(O)c(-c3ccc(I)cc3)oc2c1</smiles>

Kaempferol (18)

Figure 4. Structures of proposed flavonoids (8, 16-18). 
<smiles>CC(C)=CCC(O)C(C)=CCO[C@@H]1O[C@H](CO)[C@@H](C)[C@H](O)[C@H]1O</smiles>

Rosiridin (14)<smiles>C/C(=C\CO[C@@H]1O[C@H](CO[C@H]2OC[C@@H](O)[C@H](O)[C@H]2O)[C@@H](C)[C@H](O)[C@H]1O)CCCC(C)(C)O</smiles>

Rhodioloside E (10)

Figure 5. Structures of proposed monoterpene alcohols $(\mathbf{1 0}, \mathbf{1 4})$.

\subsubsection{Quantification of Characteristic Phenolics}

The phenylpropanoid salidroside, as well as phenylethanoids cinnamyl alcohol, its glycosides rosarin, rosavin and rosin, and flavonols rhodionidin, rhodiosin and rhodionin, were quantified by comparing peak areas in UHPLC chromatographic profiles of different extracts with those of identical or structurally similar reference standards of known concentrations. Differences in molecular weight between reference substances and analytes were considered when necessary and calculated accordingly by multiplying with correction factors (Tables 3 and 4) to give accurate quantities of investigated compounds.

Table 3. Correction factors for quantification of cinnamyl alcohol derivatives relative to reference standard.

\begin{tabular}{ccc}
\hline Cinnamyl Alcohol Derivatives & M (g/mol) & Factor \\
\hline${\text { Cinnamyl alcohol }{ }^{1}}_{\text {Rosarin }}$ & 134.17 & - \\
Rosavin & 428 & 3.190 \\
Rosin & 428 & 3.190 \\
& 296 & 2.206 \\
\hline
\end{tabular}

Table 4. Correction factors for quantification of flavonoid derivatives relative to reference standard.

\begin{tabular}{ccc}
\hline Flavonoid Derivatives & M (g/mol) & Factor \\
\hline Kaempferol-7-neohesperoside & 612.53 & - \\
$\mathrm{H}_{2} \mathrm{O}^{1}$ & 610 & 0.996 \\
Rhodionidin & 610 & 0.996 \\
Rhodiosin & 448.38 & 0.732 \\
Rhodionin & ${ }^{1}$ Reference standard.
\end{tabular}

The quantity of compounds is given as $\mathrm{mg} / 100 \mathrm{~g}$ freeze-dried plant material for ASE extracts and $\mathrm{mg} / 100 \mathrm{~g}$ fresh plant material for macerates in Table 5 for collection site High Tauern and Table 6 for collection site Gurktal Alps. The total content of salidroside and cinnamyl alcohol, as well as calculated approximative total contents of cinnamyl alcohol glycosides and flavonoid derivatives, are summarized again in Tables 7 and 8 for both collection sites, respectively. 
Table 5. Quantitative analysis of Rhodiola rosea rhizome extracts from collection site High Tauern (mg/100 g plant material $\left.{ }^{1}\right)$.

\begin{tabular}{|c|c|c|c|c|c|c|c|c|}
\hline Extracts High Tauern & Salidroside & Rosarin & Rosavin & Rosin & Cinnamyl Alcohol & Rhodionidin ${ }^{6}$ & Rhodiosin $^{6}$ & Rhodionin $^{6}$ \\
\hline $\mathrm{A}^{2} \mathrm{ASE}$ & $300.11(0.75)$ & $439.58(2.83)$ & $1565.06(0.38)$ & -5 & $31.08(0.06)$ & $33.30(1.24)$ & 391.39 (3.36) & $93.35(2.71)$ \\
\hline $\mathrm{B}^{3}$ ASE & $212.70(0.10)$ & $361.72(0.51)$ & $1389.24(0.82)$ & $34.93(4.06)$ & $53.12(0.84)$ & $80.98(0.61)$ & $352.26(1.26)$ & $81.92(2.15)$ \\
\hline $\mathrm{C}^{4}$ ASE & $237.37(0.13)$ & $251.16(2.57)$ & $1010.22(0.16)$ & $27.98(2.66)$ & $75.17(1.80)$ & $59.94(0.80)$ & $460.01(0.86)$ & $88.79(2.56)$ \\
\hline A 38\% Ethanol & 119.94 & 71.33 & - & 8.03 & 231.47 & - & - & - \\
\hline B 38\% Ethanol & 118.64 & 57.38 & - & - & 221.16 & - & - & - \\
\hline C 38\% Ethanol & 112.22 & 48.49 & - & - & 215.76 & - & - & - \\
\hline A $70 \%$ Ethanol & 145.94 & 94.17 & 138.17 & 16.75 & 147.11 & 2.60 & 19.81 & 5.61 \\
\hline B 70\% Ethanol & 109.93 & 77.65 & 97.25 & 11.35 & 158.07 & 4.34 & 10.76 & 2.35 \\
\hline C $70 \%$ Ethanol & 86.51 & 81.74 & 70.22 & 18.80 & 162.45 & 4.29 & 22.23 & 5.03 \\
\hline A $96 \%$ Ethanol & 126.79 & 93.69 & 204.74 & 28.39 & 88.76 & 3.82 & 25.90 & 5.12 \\
\hline B 96\% Ethanol & 127.89 & 77.00 & 152.84 & 30.46 & 123.32 & 7.14 & 14.55 & 2.23 \\
\hline C $96 \%$ Ethanol & 118.85 & 79.19 & 84.89 & 25.98 & 182.23 & 7.91 & 21.92 & 3.67 \\
\hline
\end{tabular}

1 per $100 \mathrm{~g}$ freeze-dried plant material in ASE, per $100 \mathrm{~g}$ fresh plant material in macerates; ${ }^{2}$ Sample A collected before flowering season; ${ }^{3}$ Sample B collected during flowering season; ${ }^{4}$ Sample C collected after flowering season; ${ }^{5}$ not quantifiable; ${ }^{6}$ quantified as kaempferol-7-O-glycosides; relative standard deviations are given in parentheses.

Table 6. Quantitative analysis of Rhodiola rosea rhizome extracts from collection site Gurktal Alps (mg/100 g plant material ${ }^{1}$ ).

\begin{tabular}{|c|c|c|c|c|c|c|c|c|}
\hline Extracts Gurktal Alps & Salidroside & Rosarin & Rosavin & Rosin & Cinnamyl Alcohol & Rhodionidin ${ }^{6}$ & Rhodiosin $^{6}$ & Rhodionin $^{6}$ \\
\hline $\mathrm{D}^{2} \mathrm{ASE}$ & $297.59(1.68)$ & 453.54 (1.93) & $1585.28(0.08)$ & -5 & $17.25(1.38)$ & $74.20(0.27)$ & $619.65(1.25)$ & $175.63(4.39)$ \\
\hline $\mathrm{E}^{3} \mathrm{ASE}$ & $402.38(0.17)$ & $364.50(2.17)$ & $737.94(0.68)$ & $40.24(1.27)$ & $159.36(0.34)$ & $141.48(0.36)$ & $320.38(1.47)$ & $79.54(2.71)$ \\
\hline $\mathrm{F}^{4} \mathrm{ASE}$ & $293.97(0.64)$ & $467.10(0.50)$ & $1552.58(1.33)$ & 41.97 (3.73) & 36.89 (1.05) & $77.50(0.53)$ & $587.41(1.58)$ & $131.66(2.11)$ \\
\hline D 38\% Ethanol & 119.74 & 80.04 & - & 12.91 & 208.92 & - & 8.72 & 1.55 \\
\hline E 38\% Ethanol & 154.68 & 46.19 & - & 2.34 & 178.81 & - & - & - \\
\hline F 38\% Ethanol & 109.58 & 57.19 & - & - & 167.71 & - & - & - \\
\hline D 70\% Ethanol & 131.20 & 95.47 & - & 14.27 & 188.60 & - & 15.12 & 3.14 \\
\hline E 70\% Ethanol & 143.24 & 73.42 & 56.49 & 13.63 & 125.47 & 5.88 & 14.52 & 2.86 \\
\hline F 70\% Ethanol & 142.14 & 76.15 & 13.87 & - & 172.29 & - & - & - \\
\hline D 96\% Ethanol & 108.93 & 104.45 & - & 21.95 & 202.48 & 6.69 & 28.46 & 4.17 \\
\hline E 96\% Ethanol & 163.77 & 69.75 & 60.21 & 25.27 & 121.25 & 9.00 & 14.45 & 1.75 \\
\hline F 96\% Ethanol & 185.14 & 85.85 & 50.40 & 14.07 & 155.96 & - & - & - \\
\hline
\end{tabular}

1 per $100 \mathrm{~g}$ freeze-dried plant material in ASE, per $100 \mathrm{~g}$ fresh plant material in macerates; ${ }^{2}$ Sample D collected before flowering season; ${ }^{3}$ Sample E collected during flowering season;

${ }^{4}$ Sample F collected after flowering season; ${ }^{5}$ not quantifiable; ${ }^{6}$ quantified as kaempferol-7-O-glycosides; relative standard deviations are given in parentheses. 
Table 7. Total contents for investigated substance groups for extracts from collection site High Tauern $\left(\mathrm{mg} / 100 \mathrm{~g}\right.$ plant material $\left.{ }^{1}\right)$.

\begin{tabular}{ccccc}
\hline $\begin{array}{c}\text { Extracts High } \\
\text { Tauern }\end{array}$ & Salidroside & $\begin{array}{c}\text { Cinnamyl Alcohol } \\
\text { Glycosides }\end{array}$ & Cinnamyl Alcohol & $\begin{array}{c}\text { Flavonoid } \\
\text { Derivatives }\end{array}$ \\
\hline A $^{2}$ ASE & 300.11 & 2004.64 & 31.08 & 518.04 \\
B $^{3}$ ASE & 212.70 & 1785.89 & 53.12 & 515.15 \\
C $^{4}$ ASE & 237.37 & 1289.36 & 75.17 & 608.74 \\
A 38\% Ethanol & 119.94 & 79.36 & 231.47 & -5 \\
B 38\% Ethanol & 118.64 & 57.38 & 221.16 & - \\
C 38\% Ethanol & 112.22 & 48.49 & 215.76 & - \\
A 70\% Ethanol & 145.94 & 249.09 & 147.11 & 28.01 \\
B 70\% Ethanol & 109.93 & 186.25 & 158.07 & 17.45 \\
C 70\% Ethanol & 86.51 & 170.76 & 162.45 & 31.55 \\
A 96\% Ethanol & 126.79 & 326.82 & 88.76 & 34.84 \\
B 96\% Ethanol & 127.89 & 260.30 & 123.32 & 23.92 \\
C 96\% Ethanol & 118.85 & 190.06 & 182.23 & 33.49 \\
\hline
\end{tabular}

${ }^{1}$ per $100 \mathrm{~g}$ freeze-dried plant material in ASE, per $100 \mathrm{~g}$ fresh plant material in macerates; ${ }^{2}$ Sample A collected before flowering season; ${ }^{3}$ Sample B collected during flowering season; ${ }^{4}$ Sample C collected after flowering season;

${ }^{5}$ not quantifiable; ${ }^{6}$ quantified as kaempferol-7-O-glycosides.

Table 8. Total contents for investigated substance groups for extracts from collection site Gurktal Alps $\left(\mathrm{mg} / 100\right.$ g plant material $\left.^{1}\right)$.

\begin{tabular}{ccccc}
\hline $\begin{array}{c}\text { Extracts Gurktal } \\
\text { Alps }\end{array}$ & Salidroside & $\begin{array}{c}\text { Cinnamyl Alcohol } \\
\text { Glycosides }\end{array}$ & Cinnamyl Alcohol & $\begin{array}{c}\text { Flavonoid } \\
\text { Derivatives }\end{array}$ \\
\hline D $^{2}$ ASE & 297.59 & 2038.82 & 17.25 & 869.47 \\
E $^{3}$ ASE & 402.38 & 1142.68 & 159.36 & 541.40 \\
F $^{4}$ ASE & 293.97 & 2061.65 & 36.89 & 796.57 \\
D 38\% Ethanol & 119.74 & 92.95 & 208.92 & 10.27 \\
E 38\% Ethanol & 154.68 & 48.53 & 178.81 & -5 \\
F 38\% Ethanol & 109.58 & 57.19 & 167.71 & - \\
D 70\% Ethanol & 131.20 & 109.74 & 188.60 & 18.26 \\
E 70\% Ethanol & 143.24 & 143.54 & 125.47 & 23.25 \\
F 70\% Ethanol & 142.14 & 90.02 & 202.48 & - \\
D 96\% Ethanol & 108.93 & 126.40 & 121.25 & 39.32 \\
E 96\% Ethanol & 163.77 & 155.23 & 155.96 & 25.21 \\
F 96\% Ethanol & 185.14 & 150.32 & - \\
\hline
\end{tabular}

${ }^{1}$ per $100 \mathrm{~g}$ freeze-dried plant material in ASE, per $100 \mathrm{~g}$ fresh plant material in macerates; ${ }^{2}$ Sample D collected before flowering season; ${ }^{3}$ Sample E collected during flowering season; ${ }^{4}$ Sample F collected after flowering season;

${ }^{5}$ not quantifiable; ${ }^{6}$ quantified as kaempferol-7-O-glycosides.

\subsubsection{Collection Site High Tauern}

Methanolic ASE proved to be more effective than any ethanolic maceration in extracting salidroside, cinnamyl alcohol glycosides and flavonoids. The highest amounts of salidroside were found in sample A (before flowering season) at $300.11 \mathrm{mg} / 100 \mathrm{~g}$ freeze-dried rhizome. Cinnamyl alcohol glycosides also reached their highest concentration in sample A, followed by B (in flowering season) and C (after flowering season). Rosavin was the peaking glycoside at $1565.06 \mathrm{mg} / 100 \mathrm{~g}$ in sample A; rosin could not be quantified in this sample. Quantities align as rosavin > rosarin > rosin across all samples. Cinnamyl alcohol was extracted in lowest quantities, with the least amount at $31.08 \mathrm{mg} / 100 \mathrm{~g}$ in sample A. An indirect relation between the amount of cinnamyl alcohol glycosides and cinnamyl alcohol could be suggested. Flavonoids were also found in highest quantities in ASE, peaking at $608.74 \mathrm{mg} / 100 \mathrm{~g}$ in sample C. Composition showed to be rhodiosin > rhodionin > rhodionidin in all samples.

In ethanolic macerates, the highest content of salidroside was found in sample A extracted with $70 \%$ ethanol at $145.94 \mathrm{mg} / 100 \mathrm{~g}$ fresh rhizome, the lowest quantity was $86.51 \mathrm{mg} / 100 \mathrm{~g}$ in sample $\mathrm{C}$ extracted with the same solvent. Highest quantity of cinnamyl alcohol glycosides was found in 
sample A extracted with 96\% ethanol at $326.82 \mathrm{mg} / 100 \mathrm{~g}$, lowest in sample C, 38\% ethanol macerate, at $48.49 \mathrm{mg} / 100 \mathrm{~g}$. Sample A > B > C and 96\% ethanol > 70\% > 38\% was true for cinnamyl alcohol glycoside content. The highest individual glycoside was rosavin in sample A macerated with $96 \%$ ethanol at $204.74 \mathrm{mg} / 100 \mathrm{~g}$. In 38\% ethanol extracts no rosavin was found, rosin only within sample A. In $96 \%$ ethanol extracts, quantities follow the same composition as ASE, with rosavin $>$ rosarin $>$ rosin. An indirect trend between cinnamyl alcohol and cinnamyl alcohol glycosides could be determined in most samples, with higher cinnamyl alcohol glycoside content relating to lower cinnamyl alcohol quantities, also quantities of cinnamyl alcohol were mostly lower in extracts of higher alcohol content. This could be due to higher rate of hydrolysis of glycosides in extracts with higher water content. The highest flavonoid content correlated with highest alcohol content, no flavonoids were found in $38 \%$ alcohol macerates. Among individual flavonoids, rhodiosin peaked at $25.90 \mathrm{mg} / 100 \mathrm{~g}$ in sample A extracted with $96 \%$ ethanol. Overall, the quantity of flavonoids was still comparatively low in ethanolic extracts.

\subsubsection{Collection Site Gurktal Alps}

ASE extraction with methanol was more efficient than ethanolic maceration for all substances except cinnamyl alcohol. Salidroside peaked at $402.38 \mathrm{mg} / 100 \mathrm{~g}$ freeze-dried rhizome in sample E (in flowering season). The highest quantity of cinnamyl alcohol glycosides was found in sample $\mathrm{F}$ (after flowering season), followed closely by D (before flowering season), and was lowest in sample E. Among individual glycosides, rosavin had the highest concentration at $1585.28 \mathrm{mg} / 100 \mathrm{~g}$ in sample D; rosin was not found in this sample. Distribution was rosavin $>$ rosarin $>$ rosin in all samples. High cinnamyl alcohol glycoside concentration again occurred with a low quantity of cinnamyl alcohol. Sample E showed a remarkably high content of cinnamyl alcohol and a low quantity of cinnamyl alcohol glycosides. This could most likely be attributed to extensive hydrolysis during transport of plant material before ASE extraction. Sample D showed highest flavonoid concentration of all investigated extracts at $869.47 \mathrm{mg} / 100 \mathrm{~g}$.

Among ethanolic extracts, the highest salidroside content was found in sample F extracted with $96 \%$ ethanol at $185.14 \mathrm{mg} / 100 \mathrm{~g}$ fresh rhizome, and the lowest was found at $108.93 \mathrm{mg} / 100 \mathrm{~g}$ in sample D extracted with the same solvent. Cinnamyl alcohol glycosides peaked at $155.23 \mathrm{mg} / 100 \mathrm{~g}$ in the $96 \%$ ethanol macerate of sample E, while its lowest quantity was $90.02 \mathrm{mg} / 100 \mathrm{~g}$ in sample F extracted with $70 \%$ ethanol. The highest individual glycoside was rosarin in the $96 \%$ ethanol macerate of sample $\mathrm{D}$ at $104.45 \mathrm{mg} / 100 \mathrm{~g}$. Again, no rosavin was found in 38\% ethanolic extracts; it was also missing in sample D extracted with $70 \%$ and $96 \%$ ethanol. Rosin could not be quantified in 38\% and $70 \%$ ethanolic macerates of sample F. Overall, there was a much higher variation in salidroside and cinnamyl alcohol glycoside contents among extracts and also no distinct correlation between cinnamyl alcohol glycoside and cinnamyl alcohol content could be recognized. Highest flavonoid content was found in sample D, $96 \%$ ethanol macerate, at $39.32 \mathrm{mg} / 100 \mathrm{~g}$. A higher quantity of extracted flavonoids again correlated with higher ethanol content of the solvent, but it was still comparatively low; no flavonoids at all could be quantified in sample E extracted with $38 \%$ ethanol and all ethanolic extracts of sample F.

\section{Discussion}

In this study we set out to analyze different preparations of Rhodiola rosea, extracted by methanolic ASE and ethanolic maceration. In qualitative analysis, 18 substances were tentatively identified by interpreting UHPLC-ESI-DAD-MS ${ }^{n}$ chromatographic data and comparing these with substances previously found in Rhodiola rosea. Among those, two cyanogenic glycosides, three phenylethanoids, two gallic acid derivatives, five phenylpropanoids, two monoterpene alcohols and four flavonols could be determined. In quantitative analysis, we measured the content of salidroside, cinnamyl alcohol glycosides rosarin, rosavin, and rosin, cinnamyl alcohol and flavonol glycosides rhodionidin, rhodiosin and rhodionin in relation to reference standards of known concentrations. 
Methanolic ASE proved to be more efficient than ethanolic maceration and produced the highest yield in all investigated substances, except for cinnamyl alcohol. This was due to the content of cinnamyl alcohol glycosides being inversely correlated to the amount of cinnamyl alcohol in the extract. As salidroside and cinnamyl alcohol glycosides are thought to be responsible for the pharmacological activity of Rhodiola rosea extracts [13], a high content of cinnamyl alcohol could be considered an unfavorable trait for preparations of the plant, although it might be reasoned that glycosides are mostly transformed and absorbed in their aglycone form after oral consumption. Overall, ASE can be recommended for production of extracts for medicinal use. Across all ASE extracts, cinnamyl alcohol glycoside composition showed to be rosavin $>$ rosarin $>$ rosin. This was previously reported for underground parts of wild-grown Rhodiola rosea from Bulgaria [31]. Concerning ethanolic maceration, $96 \%$ ethanol proved to be the preferable solvent to achieve higher content of salidroside, cinnamyl alcohol glycosides and flavonoids, although variation was high between extracts, especially for salidroside. Hydrolysis of cinnamyl alcohol glycosides occurred less in most extracts of higher ethanol content. Flavonoids were extracted in very low quantities compared to methanolic ASE, which could be due to complexation and hydrolysis processes during long-lasting extraction through maceration.

Comparison of extracts from the two collection sites High Tauern and Gurktal Alps showed great variation in substance quantities. The highest individual salidroside contents were found in the ASE extract of sample E and $96 \%$ ethanolic extract of sample F, Gurktal Alps. Cinnamyl alcohol glycoside contents were higher at Gurktal Alps in ASE extracts and 38\% ethanolic macerates of samples D and F, but lower than High Tauern in all other samples. Higher flavonoid contents were found in ASE extracts and most ethanolic extracts of Gurktal Alps, except for sample F, were extraction with ethanol yielded no flavonoids in quantifiable amounts.

Trends in substance quantities across the vegetation period could be recognized in the ASE extracts of samples from High Tauern, where cinnamyl glycosides peaked before flowering season and gradually declined during and after flowering season. This is in line with previous findings for cultivated Rhodiola rosea rhizomes of Austrian origin, where highest concentrations of cinnamyl glycosides were reported at the time of initial shoot elongation [32]. The inverse was true for cinnamyl alcohol. These trends could not be confirmed by ASE samples from Gurktal Alps; furthermore, sample E (collected during flowering season) showed an unusually high content of cinnamyl alcohol and a low quantity of cinnamyl alcohol glycosides, most likely due to hydrolysis during transport and/or storage of fresh plant material. Freeze-drying of plant material before longer transportation could reduce these hydrolysis effects. As reported in earlier studies [31,32], it was found that phytochemical contents of Rhodiola rosea are highly dependent on individual plants and geographical area. Selection of plants showing favorable traits seems to be most promising for cultivation.

\section{Conclusions}

Rhodiola rosea is a great source for different substances of potential pharmacological relevance. Ethanolic maceration of fresh plant material can impede reproducibility in the preparation of Rhodiola rosea extracts and rhizomes of wild origin underly great fluctuations in quality. When necessary, high ethanol content makes for preferable solvent composition for maceration. Methanolic ASE of freeze-dried plant material greatly improves yield for different substance groups.

Author Contributions: Conceptualization, D.V. and F.B.; methodology, F.B.; validation, S.W.; formal analysis, S.W., I.T. and F.A.; investigation, S.W. and I.T.; resources D.V.; writing—original draft preparation, F.A.; writing一review and editing, F.A. and F.B.

Funding: This research received no external funding.

Acknowledgments: As Rhodiola rosea is protected for conservation in Austria, we thank the Carinthian district authorities of Feldkirchen and Spittal an der Drau for granting collecting permissions. Motions for Gurktal Alps and High Tauern were accepted with decrees FE3-NS-1921/2014 and SP3-NS-2459/2014. Furthermore, we thank Ing. Dietmar Rossmann of Biosphärenpark Nockberge for their support.

Conflicts of Interest: The authors declare no conflict of interest. 


\section{References}

1. Bykov, V.A.; Zapesochnaya, G.G.; Kurkin, V.A. Traditional and biotechnological aspects of obtaining medicinal preparations from Rhodiola Rosea L. (A review). Pharm. Chem. J. 1999, 33, 29-40. [CrossRef]

2. Ganzera, M.; Yayla, Y.; Khan, I.A. Analysis of the Marker Compounds of Rhodiola rosea L. (Golden Root) by Reversed Phase High Performance Liquid Chromatography. Chem. Pharm. Bull. 2001, 49, 465-467. [CrossRef]

3. Panossian, A.; Wikman, G.; Sarris, J. Rosenroot (Rhodiola rosea): Traditional use, chemical composition, pharmacology and clinical efficacy. Phytomedicine 2010, 17, 481-493. [CrossRef] [PubMed]

4. Saunders, D.; Poppleton, D.; Struchkov, A.; Ireland, R. Analysis of five bioactive compounds from naturally occurring Rhodiola rosea in eastern Canada. Can. J. Plant Sci. 2014, 94, 741-748. [CrossRef]

5. Battistelli, M.; de Sanctis, R.; de Bellis, R.; Cucchiarini, L.; Dachà, M.; Gobbi, P. Rhodiola rosea as antioxidant in red blood cells:Ultrastructural and hemolytic behaviour. Eur. J. Histochem. 2005, 49, 243-254.

6. De Sanctis, R.; de Bellis, R.; Scesa, C.; Mancini, U.; Cucchiarini, L.; Dachà, M. In vitro protective effect of Rhodiola rosea extract against hypochlorous acid-induced oxidative damage in human erythrocytes. BioFactors 2004, 20, 147-159. [CrossRef] [PubMed]

7. Abidov, M.; Crendal, F.; Grachev, S.; Seifulla, R.; Ziegenfuss, T. Effect of Extracts from Rhodiola rosea and Rhodiola crenulata (Crassulaceae) Roots on ATP Content in Mitochondria of Skeletal Muscles. Bull. Exp. Biol. Med. 2003, 136, 585-587. [CrossRef] [PubMed]

8. Panossian, A.; Hambardzumyan, M.; Hovhanissyan, A.; Wikman, G. The Adaptogens Rhodiola and Schizandra Modify the Response to immobilization Stress in Rabbits by Suppressing the Increase of Phosphorylated Stress-activated Protein Kinase, Nitric Oxide and Cortisol. Drug Target Insights 2007, 2, 39-54. [CrossRef]

9. Mattioli, L.; Funari, C.; Perfumi, M. Effects of Rhodiola rosea L. extract on behavioural and physiological alterations induced by chronic mild stress in female rats. J. Psychopharmacol. 2009, 23, 130-142. [CrossRef] [PubMed]

10. Van Diermen, D.; Marston, A.; Bravo, J.; Reist, M.; Carrupt, P.-A.; Hostettmann, K. Monoamine oxidase inhibition by Rhodiola rosea L. roots. J. Ethnopharmacol. 2009, 122, 397-401. [CrossRef]

11. Pooja Bawa, A.S.; Khanum, F. Anti-inflammatory activity of Rhodiola rosea- "A second-generation adaptogen". Phytother. Res. 2009, 23, 1099-1102. [CrossRef]

12. Kwon, Y.-I.; Jang, H.-D.; Shetty, K. Evaluation of Rhodiola crenulata and Rhodiola rosea for management of type II diabetes and hypertension. Asia Pac. J. Clin. Nutr. 2006, 15, 425-432. [PubMed]

13. Tolonen, A.; György, Z.; Jalonen, J.; Neubauer, P.; Hohtola, A. LC/MS/MS identification of glycosides produced by biotransformation of cinnamyl alcohol in Rhodiola rosea compact callus aggregates. Biomed. Chromatogr. 2004, 18, 550-558. [CrossRef] [PubMed]

14. Chen, X.; Liu, J.; Gu, X.; Ding, F. Salidroside attenuates glutamate-induced apoptotic cell death in primary cultured hippocampal neurons of rats. Brain Res. 2008, 1238, 189-198. [CrossRef]

15. Zhang, J.; Liu, A.; Hou, R.; Zhang, J.; Jia, X.; Jiang, W.; Chen, J. Salidroside protects cardiomyocyte against hypoxia-induced death:A HIF-1 $\alpha$-activated and VEGF-mediated pathway. Eur. J. Pharmacol. 2009, 607, 6-14. [CrossRef] [PubMed]

16. Li, H.-B.; Ge, Y.-K.; Zheng, X.-X.; Zhang, L. Salidroside stimulated glucose uptake in skeletal muscle cells by activating AMP-activated protein kinase. Eur. J. Pharmacol. 2008, 588, 165-169. [CrossRef] [PubMed]

17. Kwon, H.-J.; Ryu, Y.B.; Jeong, H.J.; Kim, J.H.; Park, S.-J. Rhodiosin, an Antioxidant Flavonol Glycoside from Rhodiola rosea. J. Korean Soc. Appl. Biol. Chem. 2009, 52, 486-492. [CrossRef]

18. Kobayashi, K.; Yamada, K.; Murata, T.; Hasegawa, T.; Takano, F.; Koga, K.; Fushiya, S.; Batkhuu, J.; Yoshizaki, F. Constituents of Rhodiola rosea Showing Inhibitory Effect on Lipase Activity in Mouse Plasma and Alimentary Canal. Planta Med. 2008, 74, 1716-1719. [CrossRef]

19. Jeong, H.J.; Ryu, Y.B.; Park, S.-J.; Kim, J.H.; Kwon, H.-J.; Kim, J.H.; Park, K.H.; Rho, M.-C.; Lee, W.S. Neuraminidase inhibitory activities of flavonols isolated from Rhodiola rosea roots and their in vitro anti-influenza viral activities. Bioorg. Med. Chem. 2009, 17, 6816-6823. [CrossRef]

20. Rodin, I.A.; Stavrianidi, A.N.; Braun, A.V.; Shpigun, O.A.; Popik, M.V. Simultaneous Determination of Salidroside, Rosavin, and Rosarin in Extracts from Rhodiola rosea by High Performance Liquid Chromatography with Tandem Mass Spectrometry Detection. J. Anal. Chem. 2012, 67, 1026-1030. [CrossRef] 
21. Ioset, K.N.; Nyberg, N.T.; van Diermen, D.; Malnoe, P.; Hostettmann, K.; Shikov, A.N.; Jaroszewski, J.W. Metabolic Profiling of Rhodiola rosea Rhizomes by ${ }^{1} \mathrm{H}$ NMR Spectroscopy. Phytochem. Anal. 2011, 22, 158-165. [CrossRef]

22. Akgul, Y.; Ferreira, D.; Abourashed, E.A.; Khan, I.A. Lotaustralin from Rhodiola rosea roots. Fitoterapia 2004, 75, 612-614. [CrossRef]

23. Tolonen, A.; Hohtola, A.; Jalonen, J. Comparison of electrospray ionization and atmospheric pressure chemical ionization techniques in the analysis of the main constituents from Rhodiola rosea extracts by liquid chromatography/mass spectrometry. J. Mass Spectrom. 2003, 38, 845-853. [CrossRef]

24. Avula, B.; Wang, Y.-H.; Ali, Z.; Smillie, T.J.; Filion, V.; Cuerrier, A.; Arnason, J.T.; Khan, I.A. RP-HPLC determination of phenylalkanoids and monoterpenoids in Rhodiola rosea and identification by LC-ESI-TOF. Biomed. Chromatogr. 2009, 23, 865-872. [CrossRef]

25. Ali, Z.; Fronczek, F.R.; Khan, I.A. Phenylalkanoids and Monoterpene Analogues from the Roots of Rhodiola rosea. Planta Med. 2008, 74, 178-181. [CrossRef]

26. Yousef, G.G.; Grace, M.H.; Cheng, D.M.; Belolipov, I.V.; Raskin, I.; Lila, M.A. Comparative phytochemical characterization of three Rhodiola species. Phytochemistry 2006, 67, 2380-2391. [CrossRef]

27. Petsalo, A.; Jalonen, J.; Tolonen, A. Identification of flavonoids of Rhodiola rosea by liquid chromatography-tandem mass spectrometry. J. Chromatogr. A 2006, 1112, 224-231. [CrossRef]

28. Ma, G.; Li, W.; Dou, D.; Chang, X.; Bai, H.; Satou, T.; Li, J.; Sun, D.; Kang, T.; Nikaido, T.; et al. Rhodiolosides A-E, Monoterpene Glycosides from Rhodiola rosea. Chem. Pharm. Bull. 2006, 54, 1229-1233. [CrossRef]

29. Rohloff, J. Volatiles from rhizomes of Rhodiola rosea L. Phytochemistry 2002, 59, 655-661. [CrossRef]

30. Pinheiro, P.F.; Justino, G.C. Structural Analysis of Flavonoids and Related Compounds-A Review of Spectroscopic Applications; Phytochemicals; A Global Perspective of Their Role in Nutrition and Health; Venketeshwer, Rao; InTech: Rijeka, Croatia, 2012; Volume 1, pp. 33-56.

31. Marchev, A.S.; Aneva, I.Y.; Koycheva, I.K.; Georgiev, M.I. Phytochemical variations of Rhodiola rosea L. wild-grown in Bulgaria. Phytochem. Lett. 2017, 20, 386-390. [CrossRef]

32. Mirmazloum, I.; Ladányi, M.; György, Z. Changes in the Content of the Glycosides, Aglycons and their Possible Precursors of Rhodiola rosea during the Vegetation Period. Nat. Prod. Commun. 2015, 10, 1413-1416. [CrossRef]

(C) 2019 by the authors. Licensee MDPI, Basel, Switzerland. This article is an open access article distributed under the terms and conditions of the Creative Commons Attribution (CC BY) license (http://creativecommons.org/licenses/by/4.0/). 